\title{
Review of the efforts of the Japanese Society of Echocardiography for coronavirus disease 2019 (COVID-19) during the initial outbreak in Japan
}

\author{
Yoshihiro Seo ${ }^{1}$ (1) Masao Daimon ${ }^{2} \cdot$ Hirotsugu Yamada $^{3} \cdot$ Nobuyuki Kagiyama $^{4,5} \cdot$ Mitsuhiko Ohta $^{6} \cdot$ Chisato Izumi $^{7}$. \\ Kazuhiro Yamamoto ${ }^{8}$. Satoshi Nakatani ${ }^{9}$. the member of the joint committee of the Education Committee, Public \\ Relations Committee, Guidelines Committee of the Japanese Society of Echocardiography
}

Received: 19 August 2020 / Revised: 19 August 2020 / Accepted: 25 August 2020 / Published online: 5 September 2020

(c) Japanese Society of Echocardiography 2020

\begin{abstract}
Under the coronavirus disease 2019 (COVID-19) pandemic, the Japanese Society of Echocardiography (JSE) has been working to protect medical staff involved in echocardiographic examinations and to prevent secondary infections caused by the examinations since mid-March 2020. This review aims to describe the footprint of the JSE's responses, focusing on the 3 months in which the initial outbreak of COVID-19 pandemic occurred in Japan. We summarized the six parts as follows: (1) the initial actions for COVID-19 of JSE, (2) JSE's actions for infection control-associated echocardiographic examinations, (3) statements from the American Society of Echocardiography during the COVID-19 pandemic and their Japanese translation by JSE, (4) making videos for explaining the practice of echocardiography during the COVID-19 pandemic, (5) attempts with the JSE members' opinions by the communication platform and surveys, and (6) efforts of final statement during the initial spread of COVID-19. We look forward that this review will help daily practices associated with echocardiography under the COVID-19 pandemic and in the future event of an unknown infectious disease pandemic.
\end{abstract}

Keywords COVID-19 $\cdot$ Pandemic $\cdot$ Echocardiography $\cdot$ Japanese society of echocardiography

\section{Introduction}

Yoshihiro Seo, Masao Daimon, Hirotsugu Yamada, Nobuyuki Kagiyama have equally contributed to this review article.

Members of the joint committee of the Education Committee, Public Relations Committee, Guidelines Committee of the Japanese Society of Echocardiography are listed in the Acknowledgement section.

Yoshihiro Seo

yo-seo@med.nagoya-cu.ac.jp

1 Department of Cardiology, Nagoya City University Graduate School of Medical Sciences, 1 Kawasumi, Mizuho-cho, Mizuho-ku, Nagoya 467-8601, Japan

2 Department of Clinical Laboratory, The University of Tokyo Hospital, Tokyo, Japan

3 Department of Community Medicine for Cardiology, Tokushima University Graduate School of Biomedical Sciences, Tokushima, Japan

4 Department of Digital Health and Telemedicine R\&D, Juntendo University, Tokyo, Japan
Under the coronavirus disease 2019 (COVID-19) pandemic, the Japanese Society of Echocardiography (JSE) has been working to protect medical staff involved in echocardiographic examinations and to prevent secondary infections caused by the examinations since mid-March 2020. This review aims to describe the footprint of the JSE's responses,

5 Department of Cardiovascular Biology and Medicine, Juntendo University, Tokyo, Japan

6 Cardiovascular Center, Toranomon Hospital, Tokyo, Japan

7 Department of Cardiovascular Medicine, National Cerebral and Cardiovascular Center, Osaka, Japan

8 Department of Cardiovascular Medicine and Endocrinology and Metabolism, Tottori University, Tottori, Japan

9 Saiseikai Senri Hospital, Osaka, Japan 
focusing on the 3 months in which the initial outbreak of COVID-19 pandemic occurred in Japan.

\section{Overview of the initial actions}

The Japanese Society of Echocardiography (JSE) actions are summarized in Fig. 1. Prior to the statement by JSE, the American Society of Echocardiography (ASE) issued an 'ASE Statement on Protection of Patients and Echocardiography Service Providers During The statement 'the 2019 Novel Coronavirus Outbreak' was published on March 17, 2020 [1]. Drs. Hirotsugu Yamada and Kenya Kusunose of the Tokushima University translated it voluntarily and published it on the website of JSE on March 23.

Meanwhile, the infection spread rapidly in Japan and there was confusion about how to prevent infection in echocardiographic laboratories. For this reason, under the leadership of President of the time Dr. Satoshi Nakatani, JSE decided to issue its statement. On April 3, the joint committee consisting of the Education Committee, the Guidelines Committee, and the Public Relations Committee was formed to deal with the issue of COVID-19. Based on the recommendations of the ASE and the World Federation of Ultrasound in Medicine and Biology (WFUMB), the joint committee published "Recommendations for the Protection of Healthcare Workers from Infection and Prevention of Nosocomial Infections Associated with Echocardiographic
Examinations in an Epidemic of New Coronavirus Infections" on April 6, less than a week after its formation.

Since then, we have tried to take prompt action, raised awareness of the risk of infection during echocardiographic examinations, announced specific action statements for infection control, and conducted a questionnaire survey to understand the current situation. The recommendations described in this article can be viewed at JSE website (https ://www.jse.gr.jp).

\section{Actions for infection control-associated echocardiographic examinations}

The environment in which echocardiographic examinations are performed is generally a confined space, with a high risk of infection due to the proximity during the examinations. In addition, transesophageal echocardiography (TEE) is a very high-risk procedure because of the dispersion of droplets and aerosols during insertion of the probes. We have tried to raise awareness of the need for risk management, which has been largely unnoticed in normal times, through the statements.

The first thing which we emphasized during the spread of the infection was to reconsider the indications and schedules for examinations, and to reduce the number of nonurgent examinations. Besides, statements for the selection of infection prevention measures and personal protective equipment (PPE) for each examination were given according to

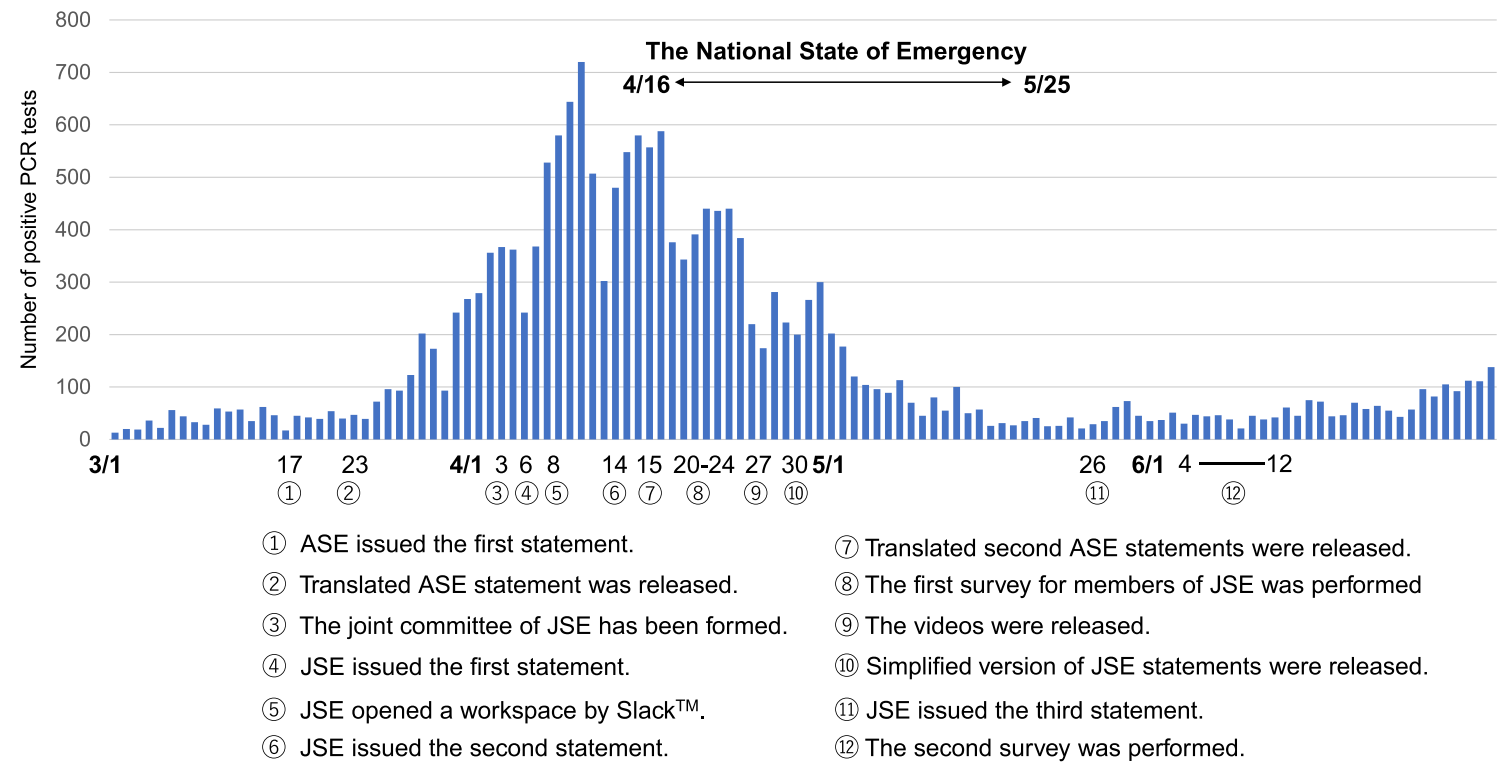

Fig. 1 Overviews of efforts of the Japanese Society of Echocardiography from March to June 2020. The graph shows the number of positive PCR tests per day in Japan. The numbers at the bottom of the graph represent dates, the circled numbers represent events, and their legends are shown at the bottom. ASE American Society of Echocardiography, JSE Japanese Society of Echocardiography, $P C R$ polymerase chain reaction 
the positive or suspected or low possibility of COVID-19, respectively (Fig. 2). The basis for this recommendation was the first statement published in March and additional statements from ASE, which were published April 6-14.

\section{ASE statements during the COVID-19 pandemic and their Japanese translation}

ASE published the first statement on the protection of patients and echocardiography service providers during the COVID-19 outbreak [1] on March 17th, 2020. At that time, although the spread of coronavirus 2 (SARS-CoV-2) infection was worried in Japan, we did not have much information about what to do in the ultrasound examination laboratory. This ASE statement provided triaging approach for prioritizing echocardiographic examinations during the COVID-19 pandemic, recommended imaging approach, and appropriate PPE use during echocardiographic examinations, which provided very useful information also for Japanese sonographers and cardiologists. Suggested algorithm for determining indication and level of protection in this statement, and its recommendations were summarized in this statement. Dr. Hirotsugu Yamada felt the necessity of the Japanese translation of this statement and made it in collaboration with Dr. Kenya Kusunose. Then, he consulted with Dr. Satoshi Nakatani, President of JSE then, about uploading the translated version to the website of the JSE. Dr. Nakatani quickly contacted ASE that night and got their permission, and the translation was uploaded on the JSE website on March 23. This was preceded by the correspondence of other societies in Japan, and could have huge influence and might dispel the anxiety of one who was involved in the ultrasound examination.
Consequently, from April 6 to 9, as supplements to the above statement about (1) pediatric, fetal, and congenital heart disease [2], (2) perioperative/periprocedural TEE [3], (3) sonographer [4], and (4) point-of-care echo (POCUS) [5] were released. Highlights of each supplements are summarized in Table 1 . The joint committee gave a request to translate these four statements, each of which was commissioned by Dr. Satoshi Yasukochi, Dr. Yoshihiro Seo, Mr. Hiroyuki Toide, and Dr.Hirotsugu Yamada. With the efforts of them and their co-authors, the Japanese translation of the four statements was completed, and after being approved by ASE, they were posted on the JSE website on April 15.

These Japanese translations also received a great response, with particular interest in infection prevention measures for examiners and ultrasound machines. However, explanations of prevention measures in the text were limited and it was thought that an explanatory video would be easier to understand. Therefore, we asked Dr. Mitsuhiko Ota (Toranomon Hospital), the representative of the Japanese Association of Young Echocardiography Fellow (JAYEF), to create a video on how to actually take these protective measures.

\section{Making videos for explaining the practice of echocardiography during the COVID-19 pandemic}

\section{Motivation for the videos}

With increasing evidence of a close interaction between cardiovascular conditions and COVID-19, there was a growing demand for echocardiography in COVID-19 patients. Since echocardiography requires physical contact between the examiner and the patient, infection of the healthcare
Fig. 2 Algorithms for indications of examination and personal protective equipment selection. This algorithm is presented in the JSE statement version 3. PPE personal protective equipment, $P O C U S$ Point-of-care Ultrasound, TEE transesophageal echocardiography, TTE transthoracic echocardiography

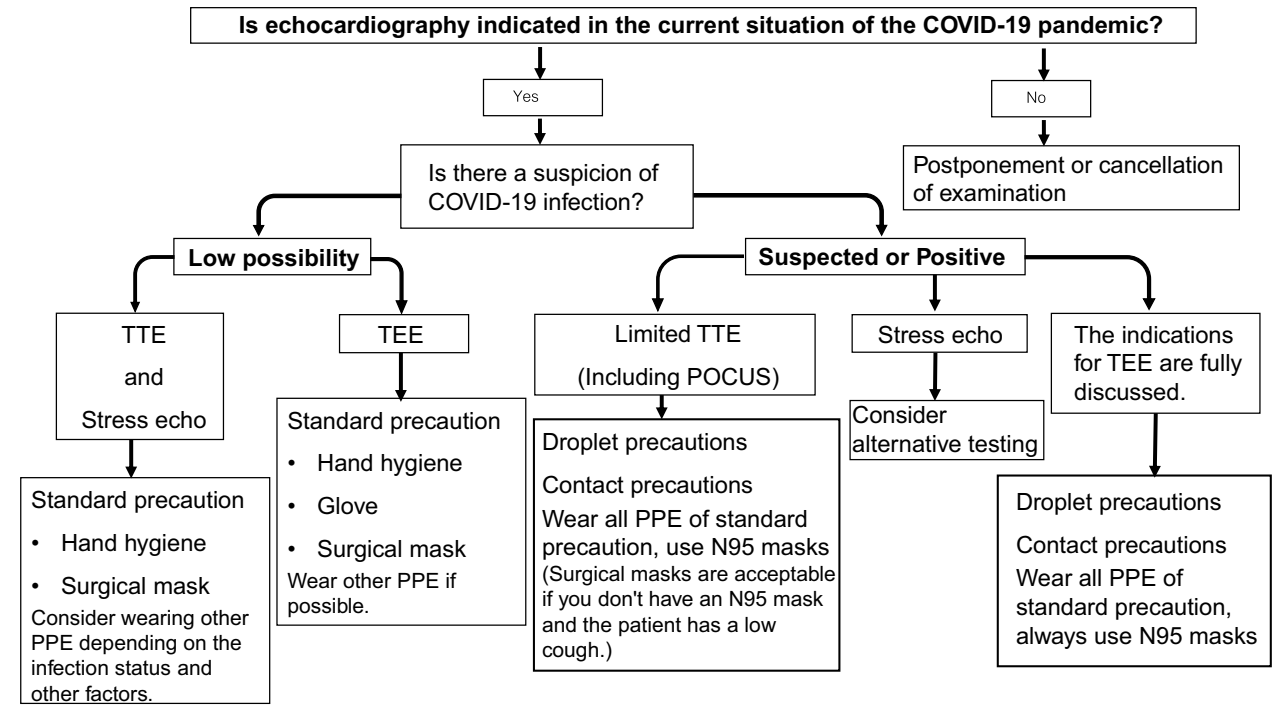


Table 1 Summary of recommendation in Supplements of ASE statement
Pediatric, fetal, and congenital heart disease patients

Children less likely to be severely affected but may be asymptomatic carriers—screening strategies' use for adults are likely to be less effective

When possible, keeping waiting and scanning areas for pregnant women/fetal echos separate from children is ideal

For low-risk patients with good cardiac imaging on routine anatomy scan, consider canceling fetal echo-specific recommendations for imaging of moderate- and high-risk patients discussed in detail in document

Consider telemedicine discussion of echo results for fetal echos-otherwise, consolidate visits as much as possible, i.e., combine with $\mathrm{OB}$ care

Given complexity of anatomy, focused TTE is likely superior to POCUS exams to limit need for repeating imaging

For intraoperative echo and all other TEE, all children without documented negative COVID testing within $72 \mathrm{~h}$ should be presumed positive

For intraoperative TEE, anesthesia should place probe immediately after intubation when full PPE is already being worn; probe should be removed while still under deep general anesthesia and cleaned immediately

Perioperative/periprocedural TEE

TEE carries a high risk for SARS-CoV-2 spread

TEE should be performed when clinical benefits outweigh the risks

Proper handling and cleaning of equipment are critical

Airborne precautions should be used in patients suspected or confirmed COVID-19

Sonographer

Sonographers need to be familiar with and prepared to implement strategies for reducing the risk of exposure to and transmission of the COVID-19 virus

Sonographers are encouraged to work with their medical teams to reschedule and/or defer all nonessential and nonemergent patients

Use of handheld devices by other trained providers may limit exposure to sonographers and conserve personal protective equipment (PPE)

In addition to the use of appropriate PPE, sonographers can use strategies that limit exposure while scanning and through appropriate cleaning of equipment

POCUS

POCUS can assist in the evaluation of suspected/confirmed COVID-19 infection

A cardiopulmonary POCUS protocol is provided, and implications discussed

A device-cleaning checklist is provided providers was the biggest concern in performing echocardiography for COVID-19 patients. Many cardiology societies, including the ASE, had thus published statements and guidelines on echocardiography for patients with COVID19, which clarify the situations where transthoracic (TTE) and TEE should be performed/avoided and when pointof-care ultrasound should be preferred. Those documents were comprehensive and extremely useful in judging the appropriateness of the examination and in acquiring and interpreting images; however, most of them were provided as written documents and were not sufficient to explain all the details in the practice. Thus, JSE sought to create videos that demonstrate the real-world practice of TTE and TEE for COVID-19 patients.

\section{Creation of videos}

First, the video for TTE was recorded at Toranomon Hospital on April 20th. In the video, an echocardiologist explained how to perform TTE in compliance with the statements from JSE published on April 14th (https://www.jse.gr.jp/COVID -JSE\%20statement2-2.pdf). The video is desired to be a guidance for echocardiographic examinations for patients with confirmed or suspected COVID-19 during the pandemic. The major points of the video are as follows.

(1) The protection of echo machines for preventing contaminations. This is important, because most echo machines involve parts that cannot be disinfected by alcohol agents, which should be disinfected using other agents that require a longer time to clean the device. The devices were covered with transparent plastic bags.

(2) Preparation of the equipment such as echo gels. They should be packed in a small disposal container, so that the gel bottles are not contaminated.

(3) Procedures in the patient's room, i.e., only image acquisition should be performed in the room and all other procedures such as data analysis should be done outside the room afterward.

(4) Disinfection of the equipment. First, the gown that the observer wore and the machine covers should be disinfected in the room. Then, immediately after leaving the room, the machine is directly disinfected with the covers removed before taking off the gown. 
(5) Sanitization of equipment in the echo laboratory such as beds, baskets, and doors.

Then, the video for TEE was created in the same manner on another day. As TEE is considered an aerosol-generating examination and poses an increased risk of the spread of the virus, TEE during the pandemic should be basically canceled or postponed unless it has a significant impact on the therapeutic strategies. However, there are cases where TEE is essential, and the video was desired to be a guidance in such situations. The contents of the video were similar as the TTE video but included some additional comments; the video stated that a very wellestablished observer should perform the examination, so that the examination time and the risk of infection are minimum; in the video, a plastic partition was placed in front of the patient for protecting the observers from droplets; the probe should be cleaned and disinfected at a central cleaning room after the examination and should not be cleaned in the department; the room should be kept air-ventilated with the door closed for a certain time (at least $3 \mathrm{~h}$ in Toranomon Hospital).

\section{Publication of the videos}

After careful evaluation by JSE core members, the videos were published on the webpage of JSE on April 27th. The videos were originally made in Japanese language; however, since they were highly appreciated and there was no other video that demonstrates the practice of echocardiography for COVID-19 patients even in other languages, JSE sought to translate the video for reaching broader audiences. Subtitles in English were attached to the videos by Dr. Nobuyuki Kagiyama (Juntendo University), so that the healthcare providers around the world are able to understand it. Fortunately, some major international academic societies and associations such as ASE and the Asian-Pacific Association of Echocardiography accepted the videos and kindly published them on their own websites (Table 2). The videos on YouTube ${ }^{\mathrm{TM}}$ by ASE have been watched more than 500 times per each.

\section{Attempts with the JSE members' opinions}

As described above, the joint committee has been proposing echocardiographic examination methods and measures to prevent the spread of the COVID-19 pandemic, but the situation was changing rapidly, and it was challenging to respond to detailed questions from the members of JSE. Then, based on the suggestion of Dr. Masao Daimon (the University of Tokyo), JSE opened a workspace on the society's website. Also, he conducted twice a survey to elucidate the status of performing echocardiography, the infection control at echocardiographic laboratory, and the needs of JSE members at that time.

\section{Information exchange by communication platform}

COVID-19 is a new infectious disease for many staff and lacks sufficient knowledge to control infection. In addition, new information about COVID-19 has been updated day by day. To accommodate these updates, we have set up a place for exchanging information through the Internet using Slack $^{\mathrm{TM}}$ (California, USA). This place is open to all JSE members, who can exchange updated information on infection status and control at any time. Questions about infection control can also be made freely by JSE members.

\section{Survey for JSE members}

From April 20 to 24, 2020, when the number of patients with COVID-19 showed the first significant increase over Japan, we conducted the first survey for members of JSE working at echocardiographic laboratory through the Internet. Subsequently, to follow the changes in these trends, a second survey was conducted from June 4 to 12 , when a tentative decrease of patients with COVID-19 was observed in Japan. A total of 609 JSE members responded to the first survey and 283 responded to the second survey. The response was mainly obtained from the areas with the high prevalence of COVID-19, in the order of Tokyo (14.9\%), Osaka (11.7\%), Hyogo (6.2\%), Kanagawa (6.2\%), and Hokkaido (4.8\%) in the first survey, and in the order of Tokyo (19.4\%), Osaka (11.7\%), Kanagawa (7.8\%), Hyogo (6.9\%), and Aichi (3.9\%)
Table 2 The webpages where the videos have been published

\begin{tabular}{ll}
\hline & Link \\
\hline JSE webpage (only Japanese versions) & https://www.jse.gr.jp/index.html \\
ASE webpage & https://www.asecho.org/covid-19-resources/ \\
YouTube by ASE (TTE) & https://www.youtube.com/watch?v=62u1-gvOyJc \\
YouTube by ASE (TEE) & https://www.youtube.com/watch?v=j_AAYwrBiMk \\
AAE webpage & https://aaecho.org/education-program/echocardio \\
& graphy-under-covid-19/ \\
\hline
\end{tabular}


in the second survey. Thus, it should be recognized that there was a regional bias in the interpretation of these surveys.

Some major results of our survey were shown in Figs. 3, 4, 5, 6. Figure 3 shows the status of performing echocardiography under the prevalence of COVID-19. In the first survey, the indication of TTE was suppressed in some ways such as postponement or cancelation in $67.2 \%$ of institutions, while it was performed as usual in $32.8 \%$ of institutions. In the second survey, the indication of TTE was still suppressed in $56.2 \%$. The indication of TEE was suppressed in $88.3 \%$ of institutions at the first survey and $89.2 \%$ of institutions at the second survey, respectively, suggesting that the prevalence of COVID-19 affected the indication of TEE more significantly, which emit aerosol. Figure 4 presents the cases to which the infection prevention measures are applied at the time of TTE at echocardiographic laboratory. This question
Fig. 3 Status of performing echocardiography. The indication of TTE was suppressed in $67.2 \%$ of institutions at the first survey and $56.2 \%$ of institutions in the second survey, respectively. The indication of TEE was suppressed in $88.3 \%$ of institutions in the first survey and $89.2 \%$ of institutions in the second survey, respectively
Fig. 4 Cases where infection prevention measures were being taken at echocardiographic laboratory. Although infection preventive measures were generally implemented in most cases at TTE, there were institutions in which no infection preventive measures were taken. Cases with definitive diagnosis of COVID-19 was excluded

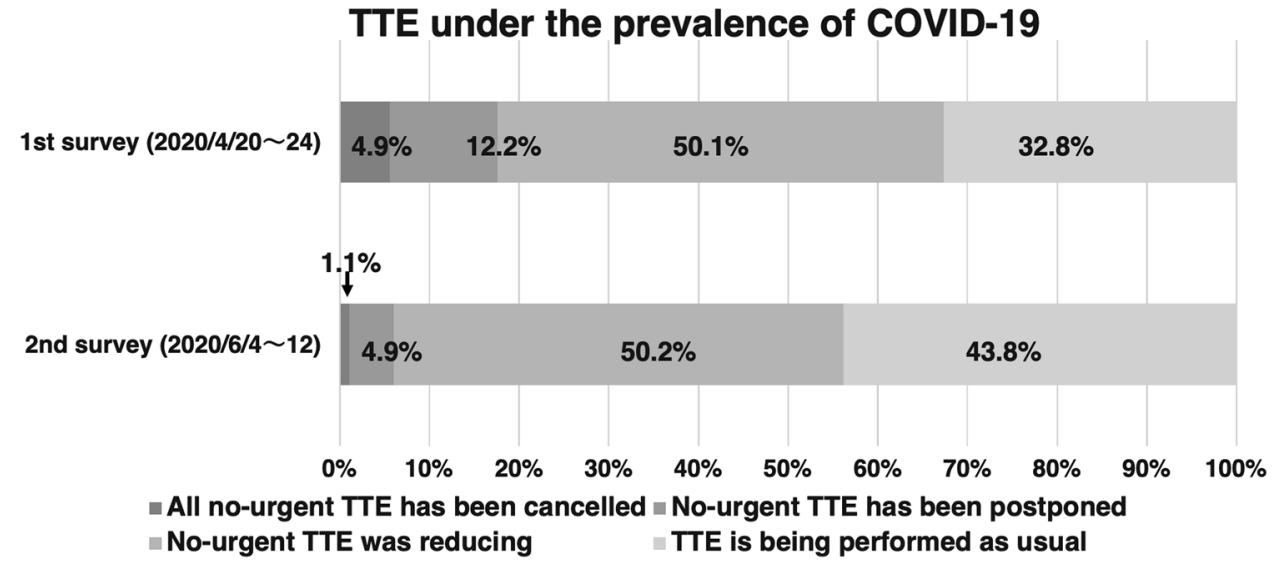

\section{TEE under the prevalence of COVID-19}
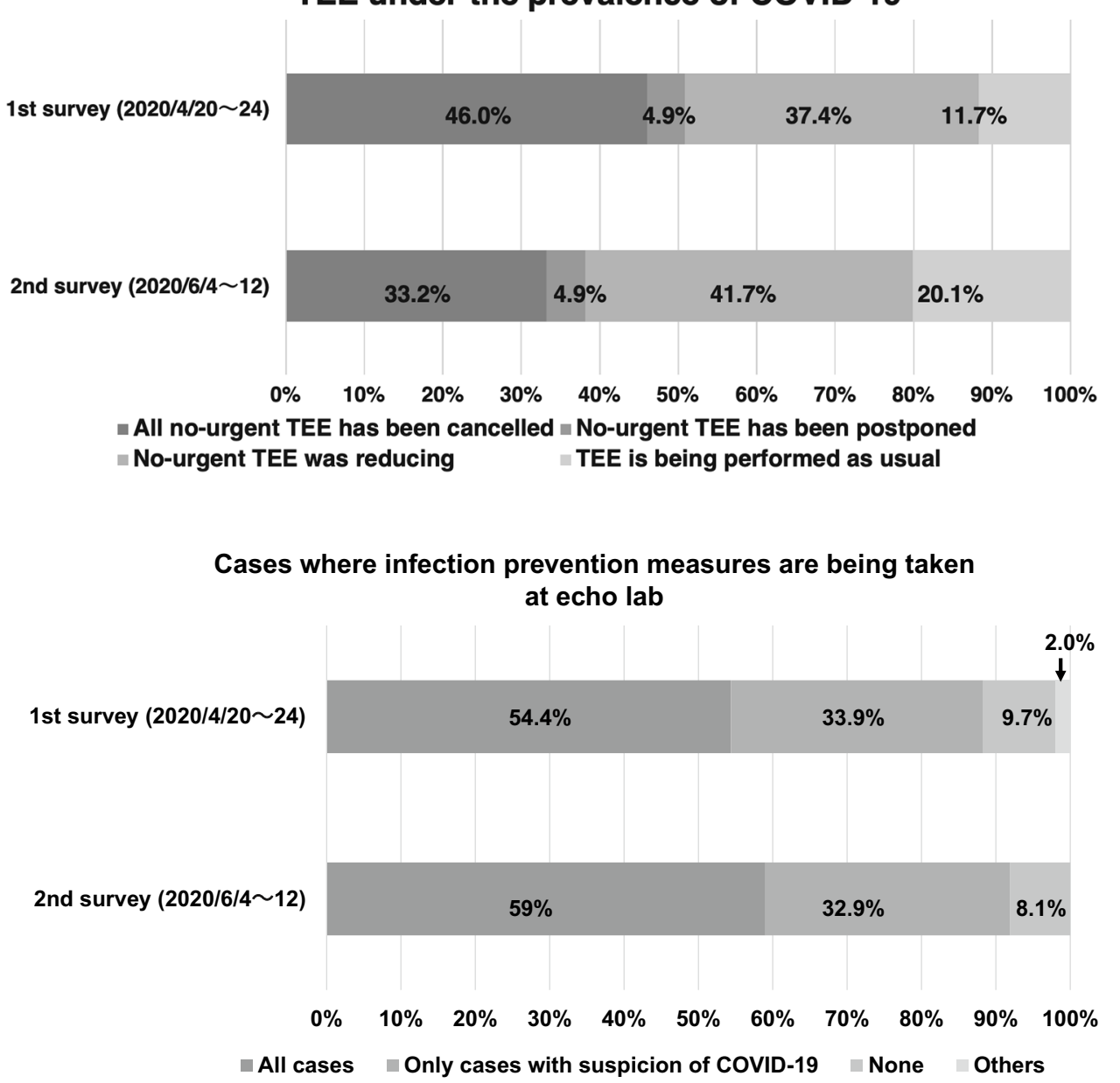
Fig. 5 Implementation rates of infection prevention measures for TTE at echocardiographic laboratory. Hand sanitizer and surgical masks were generally performed at both surveys. Implementation rates of these infection prevention measures were improved overall in the second survey. Cases with definitive diagnosis of COVID19 were excluded

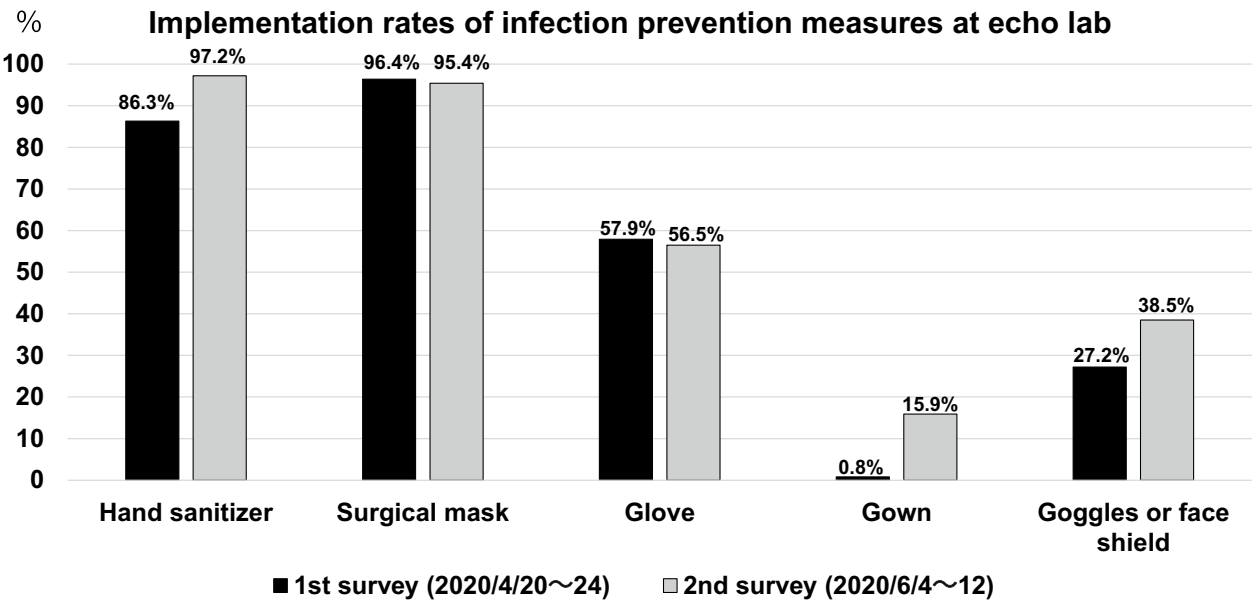

Q. Do you have Anxiety about the risk of nosocomial infection of COVID-19?

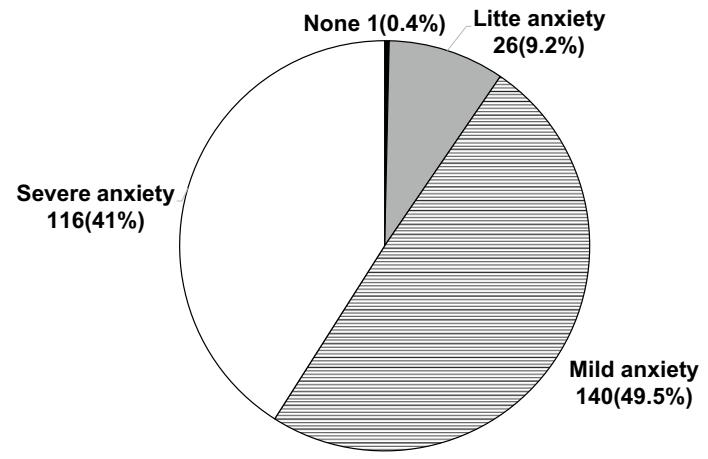

Fig. 6 Anxiety of echo staffs about the risk of nosocomial infection of COVID-19. Most responders had mild or severe anxiety about the risk of nosocomial infection of COVID-19

excludes cases that have already resulted in a definitive diagnosis of COVID-19. Although infection preventive measures were generally implemented in most cases, no infection preventive measures were taken at $9.7 \%$ in the first survey and $8.2 \%$ in the second survey. The implementation rates of infection preventive measures for TTE at echocardiographic laboratory are summarized in Fig. 5. The use of hand sanitizer and surgical masks was widely implemented, although there are some institutions where hand sanitizer was not used. Implementation rates of these infection prevention measures were improved overall in the second survey despite the decreased prevalence of COVID-19 at this time. In the second survey, we asked JSE members about their anxiety about COVID-19 infection risk (Fig. 6). More than $90 \%$ of responders working at echocardiographic laboratories had anxiety about the risk of nosocomial infection of COVID-19 (mild $49.5 \%$ or severe $41 \%$ ). In the second survey, $79.0 \%$ and $74.9 \%$ of responders were in need of "information of infection control" and "indications of echocardiography under the prevalence of COVID-19", respectively. More detailed results of the survey in Japanese are available at the JSE member portal site.

\section{Final statement during the initial spread of COVID-19}

In May, the number of infected patients has decreased, and the State of Emergency has been officially lifted for all of Japan on May 25. The number of echocardiographic examinations was expected to recover under the new routine. Meanwhile, as the number of infected patients by the second wave of CPVID-19 pandemic had been expected to increase shortly, we reiterated our statement for infection control measures on May 26, including the need for constant vigilance in medical activities against CPVID-19. In this statement, we consulted with the Japanese Society for Infection Prevention and Control (JSIPC) about PPE and infection prevention measures, so that we could explain them in detail with their help. We would like to express our sincere appreciation to the staff of JSIPC for their kind response to our questions.

\section{Conclusion}

We reviewed the JSE's response to COVID-19 pandemic from the end of March to June 2020. Through this review, it seems that the organizational capability of JSE allowed to respond to the COVID-19 pandemic with a sense of urgency. In the second survey, the majority of JSE members indicated that the Society's recommendations were useful, so that we believe that the members may have appreciated the Society's efforts. Meanwhile, we were able to collaborate with ASE on the COVID-19 pandemic response, which was a valuable experience for JSE. We will continue to monitor the current 
situation in Japan carefully and provide useful information for laboratory staff to prevent COVID-19 pandemic.

Acknowledgements We appreciate the joint committee members' efforts.

The members are as follows: Yasushi Sakata (Osaka University), Hiroyuki Toide (Dokkyo Medical University Saitama Medical Center), Kazumi Akasaka (Asahikawa Medical University), Katsuji Inoue (Ehime University), Tetsuari Ohnishi (Hyogo Brain and Heart Center), Kaoru Dohi (Mie University), Tsuyoshi Yoshimuta (Nagasaki University), Nozomi Watanabe (Miyazaki Medical Association Hospital Cardiovascular Center), Toshimi Koitabashi (Kitasato University), Takako Iino (Akita University), Kaoru Komuro (Hanaoka Seishu Memorial Hospital), Atsushi Kobayashi (Fukushima Medical University), Akihiro Hayashida (The Sakakibara Heart Institute of Okayama), Naoko Mizukami (Keiyu Hospital), Kumiko Hirata (Osaka Kyoiku University), Yasuaki Wada (Yamaguchi University), Atsushi Kotani (Kindai University Nara Hospital), Kiyohiro Takigiku (Nagano Children's Hospital), Makoto Miyake (Tenri Hospital), Hiroki Okaniwa (Gunma Prefectural Cardiovascular Center), Yutaka Hirano (Kindai University), Mitsushige Murata (Tokai University Hachioji Hospital), and Kengo Suzuki (Suzuki Medical Clinic).

\section{Compliance with ethical standards}

Conflict of interest Yoshihiro Seo, Masao Daimon, Hirotsugu Yamada, Nobuyuki Kagiyama, Mitsuhiko Ohta, Chisato Izumi, Kazuhiro Yamamoto, and Satoshi Nakatani declare that they have no conflict of interest.

\section{References}

1. Kirkpatrick JN, Mitchell C, Taub C, et al. ASE statement on protection of patients and echocardiography service providers during the 2019 novel coronavirus outbreak: endorsed by the american college of cardiology. J Am Soc Echocardiogr. 2020;33:648-53.

2. Barker PCA, Lewin MB, Donofrio MT, et al. Specific considerations for pediatric, fetal, and congenital heart disease patients and echocardiography service providers during the 2019 novel coronavirus outbreak: Council on Pediatric and Congenital Heart Disease Supplement to the Statement of the American Society of Echocardiography: endorsed by the Society of Pediatric Echocardiography and the Fetal Heart Society. J Am Soc Echocardiogr. 2020;33:658-65.

3. Nicoara A, Maldonado Y, Kort S, et al. Specific considerations for the protection of patients and echocardiography service providers when performing perioperative or periprocedural transesophageal echocardiography during the 2019 novel coronavirus outbreak: Council on Perioperative Echocardiography Supplement to the Statement of the American Society of Echocardiography endorsed by the Society of Cardiovascular Anesthesiologists. J Am Soc Echocardiogr. 2020;33:666-9.

4. Mitchell C, Collins K, Hua L, et al. Specific considerations for sonographers when performing echocardiography during the 2019 novel coronavirus outbreak: supplement to the American Society of Echocardiography Statement. J Am Soc Echocardiogr. 2020;33:654-7.

5. Johri AM, Galen B, Kirkpatrick JN, et al. ASE statement on pointof-care ultrasound during the 2019 novel coronavirus pandemic. J Am Soc Echocardiogr. 2020;33:670-3.

Publisher's Note Springer Nature remains neutral with regard to jurisdictional claims in published maps and institutional affiliations. 